\title{
Escolarizaciones intermitentes y la construcción del lugar escuela
}

Intermittent Schooling and the Construction of the School Site

Artículo de investigación | Research Article

Fecha de recepción: 20 de enero de 2020

Fecha de aceptación: 19 de enero de 2021

Fecha de disponibilidad en línea: agosto de 2021

doi: 10.11144/Javeriana.m14.eicl

Maia Acuña-Zenoff maiaacunia@hotmail.com

Universidad de Málaga, EsPaña

ORCID: https://orcid.org/0000-0001-7954-5430

Mariana Ojeda

marianaceciliaojeda@gmail.com

Universidad Nacional del Nordeste, Argentina

ORCID: https://orcid.org/0000-0003-4744-0030

SiLVIA GrinBerg

grinberg.silvia@gmail.com

Universidad Nacional de San Martín, Argentina

ORCID: https://orcid.org/0000-0001-9261-9035

Para citar este artículo | To cite this article

Acuña-Zenoff, M., Ojeda, M. \& Grinberg, S. (2021). Escolarizaciones intermitentes y la construcción del lugar escuela. magis, Revista Internacional de Investigación en Educación, 14, 1-24. doi: 10.11144/Javeriana.m14.eicl 


\title{
Resumen
}

A través de la noción de lugar escuela analizamos dinámicas escolares diarias en contextos de pobreza urbana, las cuales reflejan estrategias docentes desplegadas para ocuparse de estudiantes que, por diversos motivos, presentan asistencias discontinuas que denominamos escolaridades intermitentes. El estudio se centra en los esfuerzos realizados para hacer de la escuela un lugar de permanencia, aprendizaje y protección, dada la precariedad que atraviesa la vida barrial. Los resultados de investigación son fruto de relatos etnográficos obtenidos en una escuela secundaria pública de gestión social en la Provincia del Chaco, Argentina, que se vuelve, dentro del barrio, un espacio de enseñanza, hospitalidad y contención.

\section{Palabras clave}

Asistencia escolar; retención; fracaso escolar; enseñanza pública; escuela secundaria; abandono escolar

\begin{abstract}
Through the notion of school place we analyzed daily school dynamics in contexts of urban poverty, which reflect teaching strategies to deal with students who, for various reasons, have discontinuous attendance that we called intermittent schooling. The study focused on the ways to make the school a place of permanence, learning and protection, given the precariousness that neighborhood life provides. The research results were based on ethnographic accounts in a public secondary school of social management in the province of Chaco, Argentina that becomes, within the neighborhood, a space for teaching, hospitality and containment.
\end{abstract}

\section{Keywords}

Educational attendance; retention; academic failure; public education; secondary schools; dropping out 
Descripción del artículo | Article description

En este artículo de investigación se discuten resultados provenientes de trabajos encuadrados en el marco del proyecto Escuela secundaria y trabajo docente en el nordeste argentino. Políticas, regulaciones y actores educativos para una nueva escuela secundaria (PI - 12H001 - UNNE), de la Facultad de Humanidades, Instituto de Investigaciones en Educación, Argentina. Cabe resaltar que se trata de un equipo que desde 2003 trabaja en torno a educación secundaria y trabajo docente.

\section{Introducción}

La vida escolar ha sido, desde fines del siglo xx, interpelada de múltiples formas y frentes, ya sea señalando su inadecuación a unos nuevos tiempos, su carácter reproductivo o la necesidad de implementar cambios en las formas de enseñar, gestionar u organizar tiempos y espacios, entre muchos otros aspectos. En este marco, en el que pareciera que la escuela ha perdido su lugar, nos adentrarnos en las dinámicas del hacer escuela en su devenir diario. Para ello, retomamos los resultados de una investigación realizada en una escuela pública secundaria de gestión social en la Provincia del Chaco (Argentina), emplazada en un contexto de pobreza urbana, donde el desarrollo de alternativas pedagógicas resulta clave para ocuparse de las escolaridades intermitentes de sus estudiantes. En la densidad de la vida de esa institución, así como del barrio y de los jóvenes que concurren a ella, las preguntas por la escuela, su lugar y su centralidad adquieren especificidades que no solo reubican su tarea, sino que ofrecen elementos para pensarla como espacio construido en comunidad.

Desde hace varios decenios, a partir de la crisis del capitalismo industrial hasta la reconfiguración de los dispositivos de disciplinamiento (Foucault, 1987), nos encontramos transitando en aquello que Deleuze (1996) ha denominado sociedades de control, las cuales involucran nuevos modos del devenir de instituciones y sujetos, y donde las lógicas manageriales (Grinberg, 2008) constituyen la racionalidad propia de una era que llama a sujetos e instituciones a hacerse y realizarse a sí mismos. En muchos casos, estas lógicas han retomado las propuestas de llich (1971), asociadas con la desescolarización que el autor reclamaba en los primeros setenta, si bien muchas se plantean hoy, ya no como crítica, sino de modo propositivo'. De forma creciente, los discursos del tú puedes han ido encontrado espacio

1 Un ejemplo preciso de estos ensambles de enunciados puede encontrarse en el film "La educación prohibida". http://educacionprohibida.com/? l=es 
en la gestión del curriculum y en la configuración de las propuestas de reforma de la vida escolar, asentadas en nuevas terapéuticas del alma (Grinberg, 2008). Si bien se trata de enunciados que remiten a la escolaridad en general, en el caso que nos ocupa nos referimos a una institución y a unos estudiantes cuyas vidas resultan precarizadas, de forma tal que esta escuela constituye la única oferta educativa en un barrio compuesto por alrededor de 140 familias, afectadas por el desempleo estructural y la degradación ambiental propios de estos emplazamientos en la región y en el sur global (Grinberg, 2017). Este barrio se encuentra ubicado en una chacra, al oeste de la ciudad de Resistencia, definido por ocho manzanas, siete de ellas ocupadas por viviendas familiares y una destinada a la construcción de la escuela.

En este artículo discutimos entrevistas realizadas al equipo docente y directivo, que refieren a las maneras en que la institución se construye, procurando ocuparse de matrículas fluctuantes (Grinberg, 2009; Pérez, 2015) y escolaridades intermitentes, esto es, estudiantes que por diversos motivos y por largos periodos dejan de concurrir a la institución, pero que luego vuelven. Estas escolaridades se encuentran atravesadas por historias de vida complejas, que han llevado a la escuela a desarrollar múltiples estrategias para convertirse en la contracara de aquello que, muchas veces, deviene el mundo para estos jóvenes o, en otras palabras, buscando construir un lugar menos cruel en donde habitar (Grinberg, 2019).

Como lo discutiremos más adelante, para quienes han vivido diversas situaciones de exclusión (no solo personal, sino, también, habitacional), construir, hacer y tener lugar se vuelve algo más que un detalle. $Y$, en esa tarea, la escuela se vuelve un lugar clave. Nos centramos, así, en los debates, las acciones y las búsquedas a partir de las cuales esta institución pública, atravesada por una realidad tan compleja, construye lugar y se vuelve habitable, con el objeto de dar respuestas a dichas escolarizaciones intermitentes, cuando no, nómadas, en tanto que a veces solo dejan de concurrir por un tiempo, mientras que en otras ocasiones cambian de escuelas o de ciudad y muy al tiempo vuelven. Tal intermitencia hace que los estudiantes se encuentren en un vaivén intrincado entre la calle, el trabajo y la escuela, siendo esta última reconocida y valorada como lugar, como espacio y como soporte.

Es en este marco, como resultado del trabajo en terreno, proponemos la categoría de lugar escuela, a efectos de describir aquello que, en la institución, aparece como preocupación respecto a la generación de condiciones para que sus alumnos desarrollen un sentido de pertenencia. Si en tiempos de crisis esto vale en general para cualquier institución escolar, cuando se trata de jóvenes que en muy corto tiempo de vida han 
atravesado y atraviesan múltiples exclusiones, esta construcción de lugar se vuelve clave para propiciar una escolaridad más estable, que consiga no solo la permanencia en la escuela sino, principalmente, el aprendizaje. Cuando los estudiantes adolescentes viven vidas que se asemejan mucho a las de un adulto, que trabajan lejos de sus casas o incluso se han mudado más de una vez para conseguir empleo, lograr que sigan en la institución se vuelve un desafío del hacer diario y requiere de alternativas que puedan ocuparse de estas historias de vida signadas por la exclusión. Construir una institución capaz de proporcionar este tipo de albergue es una tarea cuya solución no solo no es sencilla, sino que reclama múltiples alternativas y acciones.

A modo de hipótesis, proponemos que la construcción del lugar escuela implica que las enseñanzas y los aprendizajes ocupen un lugar central, de forma tal que a la vez que la escuela se convierte en oportunidad de ayuda y escucha cuando los alumnos permanecen en ella, al mismo tiempo aprenden y, al hacerlo, se posibilita el despliegue de la complejidad de sus propias historias de vida.

Desde esta perspectiva, discutimos los resultados de un trabajo en terreno desarrollado durante los años 2018 y 2019, en una escuela que se fundó en Resistencia — una ciudad intermedia, capital de la Provincia del Chaco, Argentina-, a partir de las luchas de movimientos sociales por construir escuelas allí donde no había. En dicho periodo, realizamos observaciones participantes (Guber, 2014), tendientes al registro de las interacciones dentro de la escuela y del aula, de las reuniones dentro de la sala de profesores, de las ferias escolares y de los encuentros formales e informales propios de la vida escolar, tanto en las aulas como en el barrio. Las estancias en terreno nos acercaron a una escuela siempre disponible y dispuesta para construirse como un lugar habitable.

Así, tal construcción de la escuela como lugar involucra un conjunto de problematizaciones ligadas a la comprensión de la vida de los estudiantes, en la medida en que la institución se entiende como una oportunidad de vida, pero, también, como aquella que propone, busca, acciona y gestiona para que sus estudiantes puedan permanecer en su seno. Para analizar dichas problematizaciones, desarrollamos este trabajo a través de dos líneas fundamentales: 1) las discusiones, acciones y búsquedas desde las que una institución como esta, atravesada por la pobreza urbana, es construida con el objeto de volverse lugar habitable y con sentido, para proporcionar respuestas a escolarizaciones intermitentes de estudiantes mayormente excluidos del sistema escolar, y 2 ) las percepciones de cómo es vivida y sentida la docencia, a efectos de pensar la tarea de enseñanza. En este orden de ideas, proponemos que la construcción de lugar escuela supone ambos 
aspectos. A continuación, desarrollamos en un primer apartado una discusión conceptual en torno a la categoría de lugar, para luego presentar los aspectos metodológicos del trabajo, analizando en un tercer apartado las notas de campo y, finalmente, en el cuarto apartado pasar a discutirlas.

\section{Marco teórico}

La noción de lugar escuela es una categoría surgida en nuestro trabajo en terreno, construida a los efectos de permitir un acercamiento a la trama de sentidos y prácticas que atraviesan, diariamente, a las instituciones educativas emplazadas en contextos de pobreza. Consideramos que es una categoría potente, en la medida que permite reconocer las historias sobre las que cada escuela se configura, los límites entre el adentro y el afuera, la circulación de la palabra, la elaboración de propuestas pedagógicas para evitar el abandono y los modos de actuar que despliegan los docentes para dar respuestas a las múltiples demandas que se identifican en las vidas y las escolarizaciones de sus estudiantes.

Al respecto, conviene referir a investigaciones previas que han examinado la educación secundaria para jóvenes con trayectorias educativas intermitentes. En el ámbito latinoamericano, Sarcinelli (2011) expone una investigación que, desde el enfoque de la pedagogía social, trabaja con jóvenes en situación de calle en Brasil. Se trata de un estudio que pone la mirada en niños que se alejan, progresivamente, de sus hogares y que tienen un contacto intermitente o "lazos débiles y esporádicos" con sus padres y sus escuelas. En la calle consiguen trabajo y tienen contacto con la droga $y$, aunque intentan dejarla y volver a la escuela, el traspaso y la circulación entre calle, trabajo y escuela se vuelve frecuente. Se resalta el trabajo con la propia experiencia de vida de los niños, lo que se denomina el sistema niñocalle, en donde su dimensión identitaria es analizada desde el concepto de espacio en el que se mueven a diario. En ese sentido, resulta pertinente considerar esta investigación, en tanto se vincula con la idea de lugar que aquí se trabaja.

Por su parte, Salinas-Quiroz et al. (2017) profundizan la idea de "asistencias intermitentes" desde testimonios de madres uruguayas, observando que las altas tasas de movilidad residencial (especialmente en los barrios con mayores carencias de infraestructura), combinadas con los efectos culturales y actitudinales de las familias respecto a la escolarización, además de la fragilidad de hábitos que implican el cumplimiento de horarios, constituyen los principales factores de esa intermitencia. En otra vertiente, RiveroCeballos (2018) estudia las causas de la asistencia intermitente en el proceso 
de aprendizaje de niños de escuelas públicas en contextos de alta vulnerabilidad social, concluyendo que pueden ser diversas, desde problemas de salud hasta limitaciones geográficas, pero vuelve a sobresalir la posible falta de valoración de la escolarización por parte de las familias, así como la falsa condición de adultez que caracteriza sus vidas, lo que los obliga a trabajar para sumar un ingreso al hogar.

En términos de Pérez (2015), la noción de escolaridades intermitentes da cuenta de una forma de caracterizar y analizar trayectos escolares interrumpidos por "políticas de escolarización disímiles, dispersas, las cuales van conjugando, en el escenario urbano, una dinámica como espacio de lucha entre diferentes sujetos, saberes, discursos e instituciones" (p. 38). Este es un concepto que remite a movimientos de circulación en un doble sentido: por un lado, refiere a lo que hacen los estudiantes dentro del sistema educativo y, por otro, a las ausencias-presencias permanentes que caracterizan los modos de estar en la escuela (Grinberg \& Abalsamo, 2016). A ello podríamos agregar la idea de una temporalidad escolar fragmentada, debido a las formas en que los sujetos configuran su cotidianidad, esto es, a la manera en la que los jóvenes entre 13 y 18 años se debaten entre ir a la escuela o trabajar para comer y sostener una vida de adultos, como condición que les permite sobrevivir en la pobreza. Cuando pensamos en escolarizaciones intermitentes, intentamos visibilizar las vidas que transcurren en barrios "donde los sujetos quedan librados a su suerte [...] y esas formas de estar 'al borde' modulan [...] maneras particulares de habitar —y de asistir a — la escuela en contextos de fragmentación y metropolización selectiva" (Dafunchio \& Grinberg, 2013, p. 259).

En suma, si bien es posible identificar trabajos que se ocupan de los estudiantes y sus trayectorias, son pocos los que hacen eje en la vida de las escuelas, como es el caso de los mencionados en el párrafo anterior. Es en esta última línea que construimos este artículo, donde la pregunta por el lugar no solo no es un detalle que se reduce a construcciones simbólicas o a significaciones que los sujetos imprimen a la escuela, desde un sentido de habitabilidad, sino que, además, está atravesada por la necesidad de contar con aspectos materiales con los que muchas escuelas, como la de nuestro estudio, no cuentan.

Así pues, es entre lo escolar y lo barrial que los sujetos construyen sentidos materiales y simbólicos, a partir de luchas que sostienen cotidianamente. Sujetos que han ido perdiendo terreno en diferentes espacios de la vida social, pero para quienes la escuela se vuelve un lugar propio, es decir, un espacio personalmente construido. Es por ello por lo que la noción de lugar permite entender a la escuela como una locación material, en donde adquiere importancia la significación del espacio, así como su circulación, 
su inclusión y exclusión. Esto se acentúa con las dinámicas de fragmentación social y territorial que cada vez más asume la vida urbana (Grinberg \& Levy, 2009).

De este modo, compartimos con Yory-García (2007) la idea de que habitar la escuela, como locación material y simbólica, desemboca en la comprensión del habitar mismo como una teoría del lugar. Es decir que la escuela, como lugar construido para ser habitado, habla de una búsqueda en procura de cobijar un adentro y un afuera que constantemente debe problematizarse y reconfigurarse. Además, como territorio construido, la idea de lugar introduce una dialéctica de lo interno y lo externo, de lo que es y lo que hace la escuela y de aquello que queda por fuera, de lo cerrado y lo abierto, lo pequeño y lo grande, o de lo que es percibido como propio y como ajeno, como confortable o amenazante. En palabras de Viñao-Frago (1993):

[...] todo espacio es un lugar percibido. La percepción es un proceso constante. Por ello no percibimos espacios sino lugares, es decir, espacios elaborados, construidos. Espacios con significados y representaciones de espacios. Representaciones de espacios que se visualizan o contemplan, que se rememoran o recuerdan, pero que siempre llevan consigo una interpretación determinada. Una interpretación que es el resultado no solo de la percepción de la disposición material de dichos espacios, sino también de su dimensión simbólica. (p. 27)

Así, esa materialidad que la noción de lugar trae consigo no deja de portar una construcción simbólica de aquello que los sujetos hacen de sí con y en ese lugar. En esta línea, Yory-García (1993; 1997; 1998; 2001; 2005) y Tuan (2007) refieren a la noción de topofilia como una categoría que permite comprender la construcción de lugares con sentido. Dicha vinculación "no se reduce solo a espacios determinados, sino que se producen interacciones con el mundo, con el Estado y con los sujetos mismos, a la luz de pactos y acuerdos" (Yory-García, 2007, p. 2).

Desde un análisis simbólico, la noción remite a una suerte de sentimiento de apego que liga a los sujetos con lugares en los cuales, por una u otra razón, se sienten identificados y en los que se vinculan desde la afectividad. En palabras de Yory-García (2007):

La topofilia es una categoría poética del espíritu desde la cual la percepción del espacio se mediatiza, no solo por la experiencia sensible que pueda tenerse de él, sino por la fuerte carga afectiva a través de la cual se podría afirmar que este 'entra en valor', o lo que es lo mismo, en 'apropiada significación', condición que permite experimentar la idea, por ejemplo, de una institución como espacio vivido, o espacio vivenciado [...] este espacio 
habitado y vivenciado es, él mismo, su propio objeto. Se autofunda, se autogestiona y, por lo mismo, se autopertenece [...] él mismo se inaugura como discurso: el discurso (en tanto formas de habitar) que transcurre en la cotidianidad. (pp. 4-5)

Tal como lo plantea este autor, no podemos dejar de hablar de la carga afectiva que atraviesa a escuelas como la de nuestro estudio, que se han auto fundado y que se sostienen desde la autogestión de sus recursos. No se trata aquí solo de las acciones que despliegan y gestionan los docentes en la institución, sino, también, de todo aquello que forma parte de la vida y de las urgencias que se acumulan en el territorio del barrio, que no deja de ser, también, el territorio escolar.

Atender esas urgencias implica dar respuestas desde los márgenes, a través de una política popular (Gerrard, 2018), en la que la escuela se vuelve el derrotero que permite visibilizar problemas concretos, pero, también, desde la que se gestionan los recursos necesarios para que los estudiantes no se tambaleen en los bordes.

En este marco, la escuela como estructura de acogida, en términos de Bárcena \& Mélich (2014), adquiere centralidad si la pensamos como una construcción en la que la "ética de la hospitalidad" está siempre presente. Nos referimos a la idea de bienvenida al recién llegado, de apreciación del otro como radical novedad, pero, también, de la escuela como lugar en constante reconfiguración de esas identidades que llegan y que traen consigo mundos ya construidos en las esferas privadas:

En la medida en que hay que proteger al joven frente al mundo, parece que su lugar propio de maduración es la esfera privada de la familia. Su ámbito de maduración no es la esfera común de lo público. Quizá sea su destino, pero no su comienzo. Y, sin embargo, la escuela, como institución educativa de acogida, no se encuentra inserta ni en lo privado ni en lo público. Está en esa zona intermedia de lo social, cuyo auge Arendt interpreta como consecuencia negativa de la época moderna. En efecto, la escuela se aviene lugar híbrido de auge de lo social, al ser la institución que interponemos entre el campo privativo del hogar y el mundo que está ahí afuera [...] según Arendt, en su condición de recién llegados, los jóvenes necesitan de un espacio de seguridad y protección oculto a la intensa y cegadora luz del ámbito público. (Bárcena \& Mélich, 2014, pp. 86-87)

Retomando nuevamente a Viñao-Frago (1993), se trata de pensar las escuelas como puntos de significación de lo ya construido dentro de los espacios personales y sociales, ya que estas: 
[...] al fin y al cabo, como la energía, en cuanto energía, ni se crean ni se destruyen, solo se transforman. La cuestión final es si se transforman en espacios fríos, mecánicos o en espacios calientes y vivos. En espacios dominados por necesidades del orden implacable y puntos de vistas fijos, o espacios que, teniendo en cuenta lo aleatorio y un punto de vista móvil, sean antes posibilidad que límite. (p. 74)

Dicha posibilidad se convierte, prácticamente, en la única en escuelas a las que los sujetos llegan como resultado de políticas gerenciales que arrojan a su población a la exclusión, y es allí el lugar en donde esto se traduce en esas matriculas intermitentes, en la medida que son los jóvenes quienes deben hacerse cargo de sus vidas y, también, hacer valer sus derechos de acceder a la educación formal.

\section{Metodología}

El trabajo que presentamos se enmarca en el paradigma de la investigación cualitativa, específicamente en la etnografía (Chase, 2015; Rivas-Flores, 2020), dado que nos interesa profundizar en las prácticas y los relatos de los actores como un modo de acercarnos a las experiencias vividas en primera persona. Por esta razón, la obtención de información involucró largas estancias en terreno, entre los años 2018 y 2019, procurando la descripción densa (Geertz, 1989) que nos ayudó a detenernos en los detalles del hacer diario, dado que es justamente en esa densidad que encontramos elementos para comprender los significados que los sujetos imprimen a su realidad, sus acciones, prácticas y reflexiones.

A tal efecto realizamos entrevistas en profundidad al equipo directivo, a la asesora pedagógica y a docentes de diferentes asignaturas, contando con un registro de veinte voces diferentes. De dicho registro seleccionamos una muestra de seis entrevistas: las de cuatro docentes (de matemáticas, plástica, tecnología y filosofía), la asesora pedagógica y la directora, dado que tienen un vínculo de pertenencia con el barrio, es decir, son vecinos y profesionales que trabajan desde los inicios de la creación de la escuela.

Durante el trabajo de campo también planteamos entrevistas flash ${ }^{2}$ a estudiantes, vecinos del barrio y trabajadores no-docentes de la escuela, que tuvieron lugar a lo largo de los dos años de estancia en terreno, al cual

2 Se trata de entrevistas de pocos minutos, en las que se plantean preguntas sobre la vida escolar en relación con circunstancias específicas: en clases, después de una clase, en el patio o puertas afuera de la escuela. La espontaneidad de las respuestas permite recopilar aspectos que, muchas veces, exceden a la observación del investigador (Porta \& Flores, 2017). 
concurríamos dos veces por semana por las tardes, momento del día en el que funcionaba el turno del nivel secundario (desde la 1:00 p.m. hasta las 6:00 p.m.). Una vez dentro de la escuela, llevamos a cabo actividades de observación participante (Guber, 2014), tendientes, como ya se mencionó, a registrar las interacciones en el aula, en las reuniones de profesores, en ferias escolares y en encuentros formales e informales tanto de la escuela como del barrio. En ocasión de este artículo, luego de toda la construcción metodológica, resolvimos trabajar con los relatos de la directora, la asesora pedagógica y seis docentes. Asimismo, retomamos el relato de un estudiante que, en las entrevistas realizadas, refirió específicamente a las ideas que aquí se trabajan.

Las visitas al campo se desarrollaron en diferentes momentos: en el contacto previo con los informantes claves, durante las primeras visitas al campo, en los periodos prolongados en los que se interactuó con los protagonistas, recorriendo los detalles cotidianos y, finalmente, en el momento de retroalimentación con la escuela, cuando presentamos los resultados de investigación y debatimos con los docentes las categorías que emergieron del trabajo de campo.

Resulta importante aclarar aquí que la escuela secundaria de tipo pública, gratuita y de gestión social derivó de la lucha de movimientos sociales, a partir de la crisis económica y agraria que atravesó Argentina durante el año 2001, la cual golpeó, considerablemente, a regiones ya empobrecidas como la provincia del $\mathrm{Chaco}^{3}$. Durante este periodo, las condiciones de precariedad que atravesaban las vidas de miles de familias se profundizaron, esta situación desembocó en la organización colectiva, el reclamo de tierras donde construir un hogar y de una institución educativa que brindara las condiciones de acceso y derecho a la educación. En nuestro caso, hablamos de la única escuela de este barrio ubicado en la periferia de la ciudad, sin edificio propio, pero que funciona en un centro comunitario. El gobierno provincial designó ocho hectáreas para construir el barrio y el terreno en el que se levantarían los cimientos de la escuela, que, sin embargo, continúa en la espera. Frente a esta coyuntura, la importancia de construir lugar no es un dato menor.

Se trata de una escuela que cuenta con aproximadamente setenta estudiantes en el nivel secundario, en tanto que, como se ha dicho, la asistencia se presenta de manera intermitente. Los cursos que mayor cantidad

3 En el primer semestre del 2019, el nordeste argentino superó el 40\% en niveles de pobreza e indigencia. Particularmente, la Provincia del Chaco alcanzó un $42.4 \%$ y el Gran Resistencia, localidad donde se encuentra la escuela, dio como resultado un total de $46.9 \%$ de hogares que viven por debajo de la línea de la pobreza (Subsecretaría de Planificación de la provincia del Chaco, 2019). 
de alumnos tienen son los primeros (entre 25 y 30 estudiantes), mientras que los últimos pueden tener dos o tres. Quienes asisten son adolescentes que, en su mayoría, viven en el barrio o en zonas aledañas, y que se han integrado a la escuela luego de haber realizado sus trayectorias escolares en otros colegios, siempre interrumpidas por algunos de los motivos antes señalados.

Los relatos de los actores remontan a los inicios de la escuela, en 2013, momento en el que las migraciones de familias provenientes del campo hacia los centros urbanos periféricos se exacerbaron a causa de la crisis. Dadas las circunstancias, las comunidades empobrecidas comenzaron a entablar acuerdos con los gobiernos provinciales y nacionales para conseguir la designación de tierras públicas, en virtud de lo cual, tal como lo expresa el Proyecto Educativo Comunitario de la institución, la modalidad de Gestión Social nace de un acuerdo entre una organización social y política y el Ministerio de Educación, Cultura, Ciencia y Tecnología (Resolución 4759/13), siguiendo los lineamientos establecidos por la Ley de Educación Nacional N. 26 206, en sus artículos 13 y 14 y, y por la Ley de Educación Provincial N. ${ }^{\circ} 6691$, en sus artículos 4 y 23.

Sin embargo, al haber nacido como un acuerdo colectivo, el papel que cumple la escuela en el barrio es el de gestionarlo todo, en el marco de aquello que Deleuze (1996) denomina sociedad de control, la cual, mientras el Estado se coloca al margen, involucra a sujetos e instituciones haciéndose a sí mismos, agenciando recursos de manera informal a través de fondos concursales, por ejemplo, cuya evaluación es anual y no garantiza el acceso al dinero (Grinberg, 2008; 2011).

Al ser escuelas manejadas por organizaciones comunitarias, la gestión escolar tiene su base en acuerdos colectivos que involucran al barrio y funcionan a partir de un proyecto político-pedagógico que busca formar a los jóvenes, pero siempre procurando adaptarse a las realidades de la comunidad en la que viven. En este caso, se utiliza la no gradualidad como estrategia pedagógica, lo que significa que las escolarizaciones de los estudiantes no transcurren a través de grados formales y tampoco hay desaprobados o repitentes. Esto forma parte de la búsqueda por ocuparse de la intermitencia, en tanto se trabaja con cada estudiante respetando sus tiempos de aprendizaje, sus tiempos dentro de la escuela y sus capacidades. Así, los cursos de primero a quinto año son reemplazados por tres bloques (ingresante, medio y egresante), que agrupan a los estudiantes según sus conocimientos y objetivos alcanzados.

Se trata, entonces, de una escuela surgida de luchas y de movimientos sociales, en procura de construir lugar después de lo que queda cuando las crisis sociales, económicas y políticas arrasan. 
Comprender, pues, la construcción del lugar escuela requiere adentrarse en los detalles del hacer cotidiano y en las tramas institucionales que se solapan, en tanto resultados de construcciones que ocurren a tientas. Esto supone encontrar distintos espesores, lógicas y contradicciones que expresan relaciones de fuerza, poder y fuga (Grinberg, 2011; 2019).

La decisión de trabajar en esta escuela estuvo guiada por las condiciones de precariedad de la población de alumnos que atienden y por las búsquedas explícitas por parte de la escuela de alternativas para atender las escolarizaciones intermitentes. Y, si bien se trata de un trabajo que aborda la cotidianidad en escuelas emplazadas en contextos de pobreza urbana, queda la pregunta respecto a la vida de las escuelas en otros espacios de la urbe.

El trabajo en terreno que desarrollamos, como lo proponen Goetz y LeCompte (1988), no dejó de ser ecléctico, dadas las diferentes formas de obtención de datos que pueden utilizarse para acercarnos a la realidad lo más detalladamente posible. En ese sentido, se utilizó el diario de campo como una herramienta de registro que permitió contemplar los temas nodales que convergen en la vida escolar, tales como el hacer cotidiano de los docentes para atender las realidades de vidas complejas, las problematizaciones y discusiones respecto de la no gradualidad como sistema pedagógico que atiende escolarizaciones intermitentes, las búsquedas para cubrir urgencias que se presentan día a día, así como los sentidos que construyen los docentes respecto a ello.

El proceso analítico del material empírico dio inicio con la categorización de la información obtenida en las observaciones y las entrevistas. Luego se procedió a la categorización analítica del material de campo a través de la herramienta AtlasTi 8, que incluyó un trabajo en capas: por un lado, con los diferentes relatos de los informantes y, por otro, con teorías que dieron un marco a lo recolectado, además de conceptos propios o categorías nativas como, por ejemplo, la noción de lugar escuela. Finalmente, cruzamos estas fuentes para que la noción que aquí se analiza tuviera densidad y consistencia.

Para ello, el método de comparación constante (Glaser y Strauss, 1967; García, 2019) fue de gran utilidad, en la medida que nos permitió realizar un trabajo de ida y vuelta con la información recolectada, comparando los resultados con modelos ya desarrollados que, si bien no abordaban específicamente este concepto, acompañaron la construcción de una teoría con sentido propio.

En el primer cierre de campo comenzamos a dar cuenta de aquello que, en el discurso de los actores, aparecía como punto en común respecto a la preocupación por construir la escuela como un lugar que se vuelva 
habitable, logrando que los estudiantes "la entiendan y sientan como suya", tal como decía una docente. También, recurrimos a estrategias para atender las escolarizaciones de alumnos que van y vuelven porque deben trabajar para cubrir necesidades, dada la pobreza que los apremia. Ese construir lugar adquiere, entonces, especificidades nodales que discutiremos en el próximo punto.

\section{Resultados}

Lejos del discurso de crisis que envuelve constantemente al nivel secundario (Grinberg, 2009; Southwell, 2019), tratamos más bien de comprender que la escuela ha cambiado y lo seguirá haciendo conforme se desenvuelve la vida social, al igual que, más específicamente, los procesos permanentes de pauperización y crecimiento de barrios de cara a la pobreza. Es en esa realidad, signada por la precariedad y la exclusión de los estudiantes en todos los ámbitos de la vida — desde sus propias familias que no se hacen cargo de ellos, hasta el sistema educativo que los expulsa cuando completan la cantidad de faltas porque tuvieron que salir a trabajar-, que se configura esta escuela de gestión social ubicada en la periferia de la ciudad.

Importan aquí las preocupaciones, respuestas e intereses que la institución transita a los efectos de construir lugar. Con ese objeto, organizamos la discusión de campo en torno a tres categorías emergentes que dan sentido a la noción que se trabaja en este artículo: a) la permanencia de los estudiantes en la escuela y no en otro lado; b) la escolaridad cotidiana, entre negociar y marcar la cancha; y, por último, c) la circulación de la palabra.

\section{La permanencia de los estudiantes}

en la escuela y no en otro lado

Uno de los tantos días dentro de la escuela, la directora comenzó la jornada mostrándonos la planilla de asistencia, en la que llevan el control de las trayectorias de los estudiantes: "¿Ven?, están anotados cincuenta chicos, pero no están todos porque algunos se casan, tienen hijos, se van a otros lugares, o se van una o dos semanas porque consiguen alguna 'changuita' (trabajo intermitente) y vuelven". Es por ello que, como nos comentó la profesora de tecnología, resulta hasta prometedora la idea de que "el tiempo que estén dentro, antes de volver a irse, puedan sentir que tienen un lugar en el que estar incluidos y contenidos". De este modo, el mejor panorama es lograr que sientan que la escuela es su lugar, lo que en ocasiones se vincula con la posibilidad de no abandonarla y terminar la secundaria. 
La idea de lugar, como estableció la asesora pedagógica, es que a la escuela "la entiendan y sientan suya", para que las escolaridades intermitentes se tornen más estables y los estudiantes consigan no solo estar en la escuela, sino, principalmente, aprender. Así, un profesor de matemáticas nos dijo: “El primer paso es que estén adentro, solo así es que podemos empezar a trabajar y a cambiar cosas, porque si no están, no tenemos nada para hacer".

En esta misma línea, una docente explicaba que "el hecho que estén en la escuela y no en otro lado habla de algo que esta les está ofreciendo como lugar y no lo tienen fuera". Esta reflexión no deja de ser respuesta a aquello que un alumno nos narró: "Ya tenía que haber terminado la escuela pero 'pintó el amor' y cuando me vine a anotar me preguntaron por qué abandoné, les conté y me dijeron que no es conveniente dejarla y después me fui dando cuenta que nada que ver lo que estaba haciendo, ahora quiero terminar y después seguir un profesorado o algo".

Así, en esa intermitencia que no deja de afectar la vida de los estudiantes, construir el lugar escuela constituye una buena alianza, porque ambos fenómenos ofrecen la posibilidad de permanecer, de no abandonar, de continuar y terminar la escuela secundaria. Es aquí cuando aparece la pregunta por cómo se gestionan los límites entre ese estar dentro, del que hablaba la profesora de tecnología, y aquello que sucede por fuera:

Yo pienso: ¿cuáles son los límites que como escuela debemos plantearnos? Todavía lo estamos trabajando. Hay que entender que los alumnos viven realidades, muchas veces, de adultos. Hay alumnos que dejan de venir un tiempo y [luego] vuelven a venir [...] pero también el hecho que estén en la escuela no está vinculado solo a su rendimiento académico, sino a que estén ahí y no en otro lado. Por eso tenemos la jornada extendida. El que haya momentos que dejen de ir a la escuela tiene que ver con sus propias vidas que se asemejan a la de adultos, porque trabajan, porque se mudaron y viven lejos, etc. (Entrevista a la asesora pedagógica)

La jornada extendida es una estrategia que los docentes encontraron para que los estudiantes sientan la escuela como su lugar, permaneciendo desde la mañana hasta la tarde, comiendo y estudiando allí. Esta opción es parte del proyecto pedagógico, y les permite a los estudiantes estar en la escuela desde temprano, tener educación física, luego almorzar y "en el medio, tipo sándwich", según la directora, asisten a los espacios de formación profesional; una vez que están allí siguen las diferentes asignaturas, meriendan y vuelven a sus casas terminada la tarde. Pero el trabajo 
con los límites es diario, para lograr que los estudiantes estén dentro y no en otro lugar.

En ese trabajo, la escuela logra ocuparse de la intermitencia de las trayectorias, a través de la jornada extendida, por ejemplo, como una estrategia para que encuentren habitabilidad, aun sabiendo que las vidas de sus alumnos se parecen mucho a las de adultos.

Además, se procura que la escuela se vuelva espacio de permanencia, sin olvidar que el lugar escuela no es solo aquel en el que los docentes reciben a los alumnos con buena onda, sino que debe ser el ámbito en donde se juega la enseñanza y los aprendizajes. Así lo resaltó la profesora de plástica:

\begin{abstract}
Nosotros no somos tontos, que un alumno deje de venir y que, cuando vuelva, nosotros tengamos buena onda, no significa que va a aprobar la materia. Queremos lo mejor para ellos, encontrando la mejor manera. Pero no se discute, tienen que venir a clases. Lo que sí podemos discutir es si tuvieron problemas, el por qué dejaron de venir un tiempo por algo que les pasó. Siempre lo hablamos. Gustavo tuvo que dejar de venir seis meses porque se tuvo que poner a trabajar, porque no tenía para comer. Se fue a hombrear bolsas, a cargar camiones, pero es chico y no le quedó alternativa. Ahora retomó, buscó la mejor manera y a veces arañando, pero él sabe que tiene que terminar la escuela.
\end{abstract}

Vemos aquí las condiciones en las que se teje una trama entre la escuela y el mundo del trabajo, pero, también, en las que la escuela, a la vez que acompaña, procura hacer una diferencia: tenerlos dentro a través de las jornadas extendidas y el diálogo, el "siempre lo hablamos", como dice la profesora de plástica, como preocupación, como pregunta instalada y diálogo fluido. Aun así, eso no implica que cuando vuelven tienen todo aprobado, como si se hubiera estado. No se trata de castigar al que sale a hombrear bolsas, sino de acompañar, de ofrecer un espacio de hospitalidad, a quien, a veces arañando, consigue terminar la escuela. Pero ello requiere de unos límites y, como proponemos a continuación, en palabras de la asesora pedagógica, de "marcar la cancha".

\title{
Marcar la cancha
}

La escuela es el lugar al que los estudiantes asisten porque tienen derecho a aprender contenidos prioritarios y, si bien los docentes entienden las ausencias (más o menos prolongadas), también saben que es necesario "marcar la cancha", es decir, poner límites y negociar, ya que la 
construcción de la escuela como lugar tiene, ante todo, al aprendizaje como objetivo clave:

Ellos pueden usar el celular, pero si vemos que no nos están prestando atención, les decimos; ellos toman mate y tereré en las aulas. No solo se dan clases adentro, a veces abajo del árbol o en el centro del patio. Te desafían, pero porque son chicos que tienen muchos problemas en sus casas, viven solos, o están solos todo el día y eso se nota, pero vamos trabajando sobre eso, no es solo que aprendan, se agrega la cuestión de marcarles la cancha, los límites. (Entrevista a la asesora pedagógica)

Marcar la cancha es parte de una tarea que involucra la construcción del lugar escuela, un lugar que tiene que ser respetado, pero no desde lo punitivo, sino en el cual puedan crearse las condiciones para que las enseñanzas y los aprendizajes sean posibles. Construir el lugar escuela es, a la vez, marcar y delimitar, pero, también, dar clases bajo el árbol, sobrellevar los desafíos que los alumnos plantean, entender que tienen una vida que, muchas veces, transcurre en soledad y que necesitan comprender cuáles son los límites que la escuela tiene como institución formadora. Para ello es necesario negociar, limitar, de modo que puedan entender cuándo deben dejar el celular para prestar atención y cuáles son los momentos para hablar de sus problemas.

Como ya lo hemos dicho, la escuela se permite aceptar las ausencias recurrentes de los estudiantes y trabajar sobre ellas cuando regresan, ya que el proyecto pedagógico ha sido pensado desde la no gradualidad como sistema inclusivo y compensatorio. De hecho, fue "una cuestión que se planteó cuando se creó la escuela [...] para que no repitan y no existan regímenes punitivos o sanciones; que la escuela sea un espacio de libertad, pero, también, de acuerdos de convivencia" (entrevista a la profesora de plástica).

Trabajar los puntos medios entre la libertad y los acuerdos reclama múltiples modos de marcar la cancha que, también, implican "hablar una y mil veces", ampliando lo afirmado por el docente de matemáticas:

Para nosotros, el primer paso es que estén en la escuela y estando acá es que podemos empezar a trabajar y cambiar cosas, porque si no están, no tenemos nada para hacer. Este año [2019] ingresó un chico muy violento e irascible, era complicado tenerlo dentro del aula. Muchos profesores se quejaban porque no podían trabajar, pensaban en formas punitivas para abarcar esas situaciones y no lo comparto. Tenemos que hablar con él la cantidad de veces que sea necesario, bajarlo un poco, no chocar con él y que entienda que este es su lugar, entender que tiene sus tiempos, esperar que le haga el click, pero entender que es una espera no pasiva. 
Nuevamente, en ese hablar "la cantidad de veces que sea necesario" se evidencia la intención de habilitar el espacio para que el otro habite, y la preocupación de crear hábitat en el despliegue de la vida cotidiana, en los lugares de vinculación afectiva y simbólica, sin "chocar", como decía el profesor, esperando los tiempos en que la formación encuentre algún sentido para los estudiantes.

\title{
La circulación de la palabra
}

Históricamente, la escuela como institución ha delimitado jerarquías y una circulación de la palabra resguardada, exclusivamente, puertas adentro (Southwell, 2019). En nuestro caso, esta escuela funciona en un centro comunitario del barrio, por lo que se vuelve relativa, ediliciamente hablando, la idea de una delimitación de los espacios, ya que, por ejemplo, la dirección está ubicada en el patio. Tal como lo contaba una docente:

\begin{abstract}
Vos estás sentada en la mesa redonda y los estudiantes vienen y se sientan al lado, te cuentan por qué están felices, por qué están tristes, se involucran en las conversaciones entre profesores o con la directora. En toda institución hay lugares que se resguardan o en donde la información no circula para todos [...] pero acá la palabra circula libremente. Por ejemplo, la dirección es la mesa redonda que está en el centro del patio, entonces vos venís de la calle y abrís el portón y, simbólicamente, ya estás adentro de la dirección. En la mesa redonda todos tenemos voz. A mí me da la impresión de horizontalidad, de democratización, la palabra no está vedada o prohibida. (Entrevista a la profesora de filosofía)
\end{abstract}

Así, la mesa en el patio como espacio material y, también, simbólico se vuelve instancia clave para que los estudiantes encuentren un lugar de escucha y de identificación. Los lugares son compartidos y de todos, la horizontalidad en la circulación de la palabra es reconocida por los estudiantes, quienes definen sus propios espacios de debate, en el aula o en el patio, para acordar qué es lo que esperan de la escuela, pero, también, qué es lo que deben aportar para cuidarla:

[...] la libertad que tienen en la escuela ellos la manifiestan [...] el otro día hicieron una convivencia, estuvieron reunidos y discutieron cuáles son los problemas que ven en ellos y en la escuela, plantearon que quieren recuperar el compañerismo, que hay profesores que son muy académicos y no se los entiende. Hay un grupo que está dando clases afuera porque rompieron los ventiladores del salón, tiraron las mochilas al techo, entonces, plantearon que quieren recuperar su salón, que no quieren ser atacados todo el tiempo y que van a mejorar sus actitudes para cuidar lo 
que es suyo. Ellos coordinaban, fue como una asamblea, no había ningún profesor [...]. Al final nos entregaron un escrito en el que pusieron todo lo que debatieron. (Entrevista a la asesora pedagógica)

Esta escena refleja la manera en la que los estudiantes se apropian y definen sus propios espacios de circulación de la palabra (Grinberg \& Abalsamo, 2016), en los que ellos son los protagonistas y se reconocen como parte de la construcción de un proyecto educativo que anima las prácticas de inclusión escolar, además de que discuten sobre los problemas que ellos mismos generan, las consecuencias que tienen y las formas de revertir esas situaciones.

El concepto de topofilia como teoría del lugar que desarrolla YoryGarcía (2007) permite explicar la importancia que tiene la habilitación de los espacios materiales, lo construido, lo habitado, pero, también, de los significados para que la enseñanza tenga sentido. Hablamos de construcciones que dan identidad al lugar escuela: los bancos debajo del árbol, la mesa de la dirección en el centro del patio, las paredes con murales hechos por los mismos estudiantes o el pizarrón improvisado sobre un suelo de cemento. Es esto lo que, entendemos, implica construir lugar: un espacio cargado de historias y de huellas personales de quienes pisan ese suelo a diario y, en ese andar, lo hacen propio.

Pero hacer de la escuela un lugar propio se encuentra ligado, también, con la necesidad de sostener esas matrículas fluctuantes (Grinberg, 2009; Pérez, 2015), para que sea posible finalizar la secundaria en un contexto de precariedad que modula maneras particulares de asistir a ella y habitarla.

El lugar escuela permite reconocer la importancia de lo que Yory-García (2007) denomina espacios auto-fundados y auto-gestionados, en donde la horizontalidad y la circulación de la palabra funcionan como estrategias para que los estudiantes puedan reconocer actitudes y gestionar sus propios debates para no perder algo que les pertenece: el aula donde se 
dan clases, la espacialidad y un lugar que no solo se vuelve propio, sino donde también se los valora; el mismo que la sociedad, muchas veces, les ha negado.

Esta escuela fue construida desde sus cimientos por la comunidad, y en ese sentido hablamos de un lugar auto-pertenecido y auto-fundado, en donde todos son reconocidos y tienen voz. Los estudiantes encuentran algo que les pertenece, un espacio que no encontraron y les fue negado por fuera. Tienen, además, docentes que representan la autoridad, ponen límites, buscan alternativas y trabajan pedagógicamente la complejidad del presente que les toca vivir a sus alumnos.

Vemos cómo en la escuela se solapan múltiples factores que refieren a la desigualdad y se acumulan a diario. Los docentes hacen malabares con las vidas excluidas de sus estudiantes, quienes llegan como resultado de una estructura social frágil que los expulsa de los circuitos formales que ofrece el sistema educativo. De por sí, hay una fragilidad propia del contexto en el que tienen lugar sus vidas, y en esa fragilidad también nace la escuela. Nos referimos a que la misma no nace al calor de una política activa que se propone fortalecer los centros escolares en los barrios marginales, sino como resultado de los esfuerzos de la comunidad que, de manera más o menos articulada, se organiza e intenta construir un lugar cuyas condiciones permitan atender la dificultad para que los estudiantes permanezcan. La permanencia también significa que cuando ellos están allí tienen que esforzarse por aprender, aunque a veces lleguen "arañando", como lo resaltaba la asesora pedagógica.

Aquí tiene una centralidad el concepto de hospitalidad trabajado a través de Bárcena \& Mélich (2014). En tal sentido, se despliega una suerte de estructura de acogida para que las intermitencias escolares no terminen por convertirse en abandono. La escuela se vuelve espacio de hospitalidad y de contención, pero, como toda institución formal, también marca territorios y límites, y las normas terminan por establecerse desde una tensión tambaleante, ajustándose entre contar con ciertas flexibilidades (como la no gradualidad) y determinar acuerdos de convivencia. Por su parte, la no gradualidad, más que una estrategia para sostener las escolarizaciones intermitentes, se constituye como un sostén, un proyecto, una forma de concebir el tiempo de esas escolaridades puertas adentro.

\section{Conclusiones}

Este artículo representa una serie de elementos para pensar las formas en que una escuela pública emplazada en contexto de pobreza re/ construye el lugar a diario, como forma de ocuparse de las escolaridades 
intermitentes de sus estudiantes. Aunque en el campo de la investigación educativa es posible encontrar trabajos acerca de las trayectorias escolares de los estudiantes, este artículo se centró en otra mirada de la cuestión, que remite al hacer diario de la escuela y a sus construcciones cotidianas. Por ello se da cuenta de las estrategias que se despliegan para que la enseñanza y el aprendizaje ocupen un lugar clave, así como de las búsquedas de la escuela para lograr que los estudiantes permanezcan y terminen la secundaria, y, también, para que se arraiguen en un lugar que puedan sentir como suyo.

La categoría lugar escuela propuso otras dinámicas, principios y lógicas educativas, que van desde el gobierno a través de la comunidad, la gestión propia y el gerenciamiento de recursos para sostener matrículas en riesgo pedagógico. Todo ello mediante el ofrecimiento de un enfoque novedoso para la educación y las ciencias sociales, lo que supone, a su vez, reconocer el carácter educativo de los procesos de lucha, resistencia y organización comunitaria.

Se muestra en el estudio que, en las sociedades contemporáneas, crear lugar involucra una compleja tarea, no solo porque, como señalamos al inicio, la escuela se encuentra en constante cuestionamiento, sino porque las vidas precarizadas y excluidas reclaman instituciones donde se aprende y se enseñe. Sin embargo, las escuelas no dejan de estar y hacerse a sí mismas en medio de una vorágine, sumergidas en una precariedad que urge reparar, por lo que las mismas deben encontrar, sin perder el eje de la enseñanza, las formas apropiadas. Los apremios son muchos, y está claro que trascienden lo que se encuentra al alcance de las manos, teniendo que ampliar sus posibilidades de autogestión y, por tanto, viéndose enfrentadas a la incertidumbre constante.

Los sujetos llegan a ellas como resultado de políticas gerenciales que arrojan a la población a la exclusión, por lo que las escuelas terminan siendo el lugar en donde esto se traduce en matriculas intermitentes. Mientras tanto, a través de prácticas que se desenvuelven en los márgenes, la escuela deviene lugar que combina preocupación y políticas populares para abordar dichas intermitencias. Reconocerlo permite comprender las dinámicas contemporáneas propias del campo de la educación en el siglo xxı, así como el lugar que ocupa el Estado en la vida de instituciones emplazadas en contextos de extrema pobreza urbana.

En este marco, el Estado gestiona informalmente y los estudiantes, aun dentro de la escuela, continúan siendo afectados, estructural y sistemáticamente, por condiciones de alta vulnerabilidad social. Las políticas de gerenciamiento, al parecer, permiten un desligamiento sutil de estas tramas escolares, interviniendo informalmente sobre instituciones que ya 
vienen desplegando estrategias propias de adaptación para responder a sus urgencias cotidianas.

De igual modo, habría que preguntarse hasta qué punto la construcción de un lugar propio, en el que escuela y comunidad gestionan recursos y acciones, termina siendo una estrategia más, entre tantas, para sobrevivir en contextos de extrema pobreza urbana, mientras la precariedad no solo sigue estando a la orden del día, sino que se profundiza aún más cuando el Estado no interviene de manera directa. Actualmente, en tiempos de pandemia, vemos que esta situación no ha hecho más que profundizarse y la educación por venir, más allá de pantallas y virtualidad, tendrá que enfrentar la pregunta de qué hacer desde su lugar, más aún si espera darle solidez a la tarea de educar.

\section{Sobre los autores}

Maia Acuña-Zenoff es profesora en ciencias de la educación. Su investigación se centra en la labor de la docencia en tiempos de crisis y en las reconfiguraciones escolares en escuelas secundarias públicas de la provincia del Chaco. Es becaria doctoral de investigación del CONICET.

Mariana Ojeda es magíster en docencia universitaria, especialista en docencia universitaria de la Universidad Nacional del Nordeste (Argentina) y profesora licenciada en ciencias de la educación de la Facultad de Humanidades de la misma universidad.

Silvia Grinberg es doctora en educación (Universidad de Buenos Aires [UBA], Argentina), magíster en ciencias sociales (Facultad Latinoamericana en Ciencias Sociales, FLACSO) y especialista en sociología de la educación y de la pedagogía. Su investigación se centra en la desigualdad que puede observarse en el cruce entre escuela, sujetos y territorio.

\section{Referencias}

Bárcena, F. \& Mélich, J. (2014). La educación como acontecimiento ético. Natalidad, narración y hospitalidad. Paidós.

Chase, S. (2015). Investigación narrativa. Multiplicidad de enfoques, perspectivas y voces. En N. Denzin \& Y. Lincoln (comps.), Métodos de recolección y análisis de datos. Manual de investigación cualitativa (pp. 58-112). Gedisa.

Dafunchio, S. \& Grinberg, S. (2013). Biopolítica y experiencia de la escolaridad en contextos de extrema pobreza urbana y degradación ambiental. Magistro, 7(14), 245-269. https://core.ac.uk/download/pdf/159293664.pdf

Deleuze, G. (1996). Conversaciones. Pre-textos.

Foucault, M. (1987). Historia de la sexualidad, 1. La voluntad de saber. Siglo XXI. 
García, P. D. (2019). El método comparativo constante y sus potencialidades para el estudio de políticas educativas para la escuela secundaria en Latinoamérica. Revista Latinoamericana de Educación Comparada, 10(15), 27-43. https://dialnet.unirioja.es/servlet/articulo?codigo $=7075519$

Geertz, C. (1989). La interpretación de las culturas. Gedisa.

Gerrard, J. (2018). Empresa social, educación y trabajo: emprendedurismo en los márgenes. Journal of Education Policy, 34(6), 771-788.

Glaser, B. \& Strauss, A. (1967). The discovery of grounded theory: strategies for qualitative research. Aldine de Gruyter.

Grinberg, S. (2008). Educación y poder en el s. XXI. Gubernamentalidad y pedagogía en las sociedades de gerenciamiento. Ed. Miño y Dávila.

Grinberg, S. (2009). Políticas y territorios de escolarización en contextos de extrema pobreza urbana. Dispositivos pedagógicos entre el gerenciamiento y la abyección. Archivos de Ciencias de la Educación, 3(3), 81-98. http://www. memoria.fahce.unlp.edu.ar/art_revistas/pr.4084/pr.4084.pdf

Grinberg, S. (2011, agosto 8-10). Gubernamentalidad y educación en tiempos de gerenciamiento. Reflexiones en torno de la experiencia de los dispositivos pedagógicos en contextos de extrema pobreza urbana [Actas. La Plata: UNLP. FAHCE. Departamento de Ciencias de la Educación]. VIII Encuentro de Cátedras de Pedagogía de Universidades Nacionales Argentinas, La Plata.

Grinberg, S. (2017). Vivir y estudiar en las favelas del sur global: modulaciones gerenciales de las biopolíticas de la vida urbana. Educar em Revista, 33, 57-76. https://doi.org/10.1590/0104-4060.53863

Grinberg, S. (2019). Self-made school and the everyday making in Buenos Aires slums. British Journal of Sociology of Education, 40(4), 560-577. https://doi. org/10.1080/01425692.2019.1565991

Grinberg, S. \& Abalsamo, M. (2016). La escuela como espacio de lo común. Circulación y producción de la palabra y ciudadanía en escuelas secundarias emplazadas en contextos de extrema pobreza urbana del AMBA. Editorial CLACSO, 5(23), 1-47.

Grinberg, S. \& Levy, E. (2009). Pedagogía, currículo y subjetividad: entre pasado y futuro. Ed. Universidad Nacional de Quilmes.

Goetz, J. P. \& Lecompte, M. D. (1988). Etnografía y diseño cualitativo en investigación educativa. Ediciones Morata.

Guber, R. (comp.). (2014). Prácticas etnográficas. Ejercicios de reflexividad de antropólogas de campo. Instituto de Desarrollo Económico y Social (Ides)Miño y Dávila Editores.

Ilich, I. (1971). La sociedad desescolarizada. Ediciones Godot. Colección Exhumaciones.

Pérez, A. (2015). Políticas de escolarización, territorio y desigualdad educativa en épocas de gerenciamiento. Una mirada en clave territorial de la escolarización obligatoria estatal en el decenio de 2001-11. El caso de Caleta Olivia, provincia de Santa Cruz, Argentina [Tesis de maestría]. UNLu.

Porta, L. \& Flores, G. (2017). Investigación narrativa en educación: la expansión del valor biográfico. Revista del I/CE, 41, 35-46. http://revistascientificas.filo. uba.ar/index.php/iice/article/view/5156 
Rivas-Flores, I. (2020). La investigación educativa hoy: del rol forense a la transformación social. Márgenes Revista de Educación de la Universidad de Málaga, 1(1), 3-22. https://doi.org/10.24310/mgnmar.v1i1.7413

Rivero-Ceballos, V. (2018). La asistencia intermitente en el proceso de aprendizaje. Revista Enfoques, (2), 1-18. https://revistaifd.files.wordpress.com/ 2018/07/asistencia-intermitente-en-el-proceso-de-aprendizaje-v-rivero.pdf

Salinas-Quiroz, F., Silva, P., Cambón, V., \& Fraga, S. (2017). Asistencia intermitente y deserción en Educación Inicial. Testimonios de madres uruguayas. Revista Latinoamericana De Ciencias Sociales, Niñez Y Juventud, 15(2), 913-925. https://doi.org/10.11600/1692715x.1520828042016

Sarcinelli, A. (2011). Infancias marginales, los márgenes de la infancia. Trayectorias de muchachos en situación de calle en el noreste brasileño. Revista Alteridades, 21(42), 91-101. http://www.scielo.org.mx/pdf/alte/v21n42/v21n42a7.pdf Southwell, M. (2019). Escuela secundaria, convivencia y participación. Eudeba.

Tuan, Y. (2007). Topofilia. Un estudio de las percepciones, actitudes y valores sobre el entorno. Melusina.

Viñao-Frago, A. (1993). Del espacio escolar y la escuela como lugar: propuestas y cuestiones. Revista Historia de la Educación, 12(13), 17-74. https://revistas. usal.es/index.php/0212-0267/article/view/11367

Yory-García, C. M. (1993). Topofilia: una alternativa en torno a la revolución de las pequeñas cosas. Coedición del Programa de las Naciones Unidas para el Desarrollo (PNUD) y la Alcaldía Mayor de Bogotá.

Yory-García, C. M. (1997). La topofilia, una estrategia para hacer ciudad desde sus habitantes [Cuadernos de Estudios Urbanos. Construcción sociocultural del espacio urbano]. Editorial Corporación de Estudios de Antropología Urbana.

Yory-García, C. M. (1998). Topofilia o la dimensión poética del habitar. Ed. CEJAColciencias.

Yory-García, C. M. (2001). La topofilia: una estrategia innovadora de desarrollo sustentable para las grandes metrópolis latinoamericanas en el contexto de la globalización. Revista Anales de Geografía de la Universidad Complutense de Madrid, 21, 119-136. https://revistas.ucm.es/index.php/AGUC/article/ view/AGUC0101110119A

Yory-García, C. M. (2005). Ciudad y sustentabilidad II. Componentes y contenido de un proyecto sustentable de ciudad a partir del concepto de topofilia. Ed. Escala.

Yory-García, C. M., (2007). Del espacio ocupado al lugar habitado: una aproximación al concepto de topofilia. Barrio Taller, 12(13), 42-64. 